\title{
极地动物基因资源研究现状与发展战略
}

\author{
陈松林 ${ }^{1,2}$, 徐文腾 ${ }^{1,2}$, 陈张帆 ${ }^{1,2}$ \\ (1. 中国水产科学研究院黄海水产研究所, 山东青岛 266071；2. 青岛海洋科学与技术试点国家实验室 \\ 海洋渔业科学与食物产出过程功能实验室, 山东青岛 266237)
}

\begin{abstract}
摘要: 本文主要从基因组和转录组层面分析了极地动物基因资源的研究现状, 梳理了本领域研究中存在的问题, 并提出了未 来发展战略。极地动物基因组测序起步较晚, 迄今只完成了 13 种极地动物的全基因组测序。在转录组研究方面, 人们对极 地的 31 个物种进行了转录组测序, 并在以下四个方向重点开展了研究: 环境适应性研究; 污染物应激反应的分子机制研究; 不同发育阶段或不同组织中的转录组分析; 功能基因挖掘。本领域研究由于起步晚, 研究广度和深度都有待加强, 但极地动 物的基因资源研究具有战略意义。建议国家设立 “极地动物基因资源发掘与应用” 重点研发计划专项, 围绕极地渔业动物特 殊性状遗传解析、特有基因功能分析和基因工程产品研发等开展研究。
\end{abstract}

关键词: 极地动物; 基因组; 转录组; 基因资源

中图分类号：Q1 文献标识码：A

\section{Polar Animal Genetic Resources: Current Situation and Development Strategies}

\author{
Chen Songlin $^{1,2}$, Xu Wenteng ${ }^{1,2}$, Chen Zhangfan ${ }^{1,2}$ \\ (1. Yellow Sea Fisheries Research Institute, Chinese Academy of Fishery Sciences, Qingdao 266071, Shandong, China; \\ 2. Laboratory for Marine Fisheries Science and Food Production Processes, Pilot National Laboratory for \\ Marine Science and Technology (Qingdao), Qingdao 266237, Shandong, China)
}

\begin{abstract}
This article has reviewed the current status of polar animal genetic resources and proposed the development strategies based on existing problems. Genome sequencing of polar animals started late, and the whole genome sequencing has been conducted so far on only 13 polar animals. Transcriptome sequencing has been carried out for 31 polar animals in recent years, and the transcriptome research has focused on adaption to polar environments, molecular mechanisms in response to pollutant stresses, transcriptome changes during various development stages or within different tissues, and exploitation of functional genes. The late initiation in the study of polar animal genetic resources limited its current depth and width in research. However, this study is strategically important. We suggest that China set up a key research and development program "exploration and application of biological gene resources in polar animals" to support the work in this field, and focus on genetic dissection of special traits, functional analysis of specific genes, and development of genetically engineered products.
\end{abstract}

Keywords: polar animal; genome; transcriptome; genedic resources

收稿日期 : 2019-06-20; 修回日期 : 2019-08-26

通讯作者: 陈松林, 中国水产科学研究院黄海水产研究所研究员, 研究方向为水产基因资源发掘与应用; E-mail: chens1@ysfri.ac.cn 资助项目：中国工程院咨询项目“海洋强国战略研究 2035”(2018-ZD-08)

本刊网址： www.engineering.org.cn/ch/journal/sscae 


\section{一、前言}

极地的生存环境极端恶劣, 气候酷寒干燥, 食 物资源稀缺, 每年长达数月的极昼极夜使得生物节 律难以保持, 新陈代谢被打乱。极地动物是如何在 这种环境下生存? 哪些基因帮助它们调节、适应极 端寒冷和高盐等环境, 海中鱼类如何抗冻? 极地陆 上动物如何保暖? 这些都是极为有趣的科学问题。 然而, 长久以来极地以其寒冷的生存环境严重制约 了生命科学领域的研究, 近年来随着极地勘测和样 品获取技术的飞速发展, 极地动物生命科学领域的 研究也取得了长足进步。揭秘极地动物, 特别是渔 业动物的基因组结构, 发掘功能基因, 探究极地动 物适应寒冷环境的分子机制, 不仅具有重要的科学 意义，而且还有重大的应用价值和潜力。

\section{二、极地动物基因资源研究与应用现状}

\section{（一）极地动物基因组研究现状}

1. 极地动物基因组研究处于起步阶段

据不完全统计，截至 2018 年 9 月，已进行基 因组测序的动物种类有 380 余种，发表论文 385 篇 (见图 1（a)), 而现今只有 13 种极地动物的基因组 发表在 12 篇文章上, 比例不足 3\%(见表 1)。当然, 这与极地动物样品获取困难、基因组研究起步较 晚有关。

如图 1 所示, 我们可以看到动物基因组研究始
于 1998 年。以 2010 年为节点, 文章发表数量呈现 明显增加趋势, 而在 2011 年大西洋鳕鱼才是第一 个完成基因组测序的极地动物 [1]（见图 1（b)）。 此外，极地动物资源并不像想象中的那么圄乏，尽 管极地动物中哺乳动物的种类有限, 但鱼类的种类 可达数百种。因此, 相对极地动物的多样性而言, 基因组的相关研究存在明显的滞后。

2. 国外研究机构在极地基因组研究工作中占据 主导地位

除了企鹅以及近期南极美露鳕的基因组文章由 我国科学家领衔完成, 其他 10 篇极地动物基因组 文章的第一单位均来自国外：北极红点鲑、白鲸 (加拿大 2 种)、南极蠓、北极熊（美国 2 种）、大 西洋鳕鱼、大西洋鲑鱼（挪威 2 种）、革首南极鱼、 扁嘴副带腭鱼、南极桡足类、小须鲸 (韩国 4 种), 见表 1 。其中大多数是位于北美或北欧的研究机构, 这与他们的地理优势以及长期以来对极地的重视密 切相关。然而值得注意的是, 韩国已经对革首南极 鱼、扁嘴副带腭鱼、南极桡足类、小须鲸 4 个物种 进行了基因组测序，成为完成极地动物基因组测序 最多的国家（极地动物基因组工作的国别归属均以 第一完成单位所在国家为准)。

\section{（二）极地动物转录组的研究进展和现状}

极地动物具有独特的生物学现象和性状, 如抗 冻鱼的抗冻性状及其缺少血红细胞、北极露脊鲸的 长寿和不患癌症等。转录组是研究基因表达的重要

表 1 已完成基因组测序的极地动物

\begin{tabular}{lcccc}
\hline 中文名 & 拉丁名 & 基因组大小 & 作者及发表时间 & 发表刊物 \\
\hline 大西洋鳕鱼 & Gadus morhua & $830 \mathrm{Mb}$ & Star et al., 2011 & Nature[1] \\
北极熊 & Ursus maritimus & $2.53 \mathrm{~Gb}$ & Miller et al., 2012 & PNAS[2] \\
革首南极鱼 & Notothenia coriiceps & $637 \mathrm{Mb}$ & Shin et al., 2014 & Genome Biology[3] \\
小须鲸 & Balaenoptera acutorostrata & $2.76 \mathrm{~Gb}$ & Yim et al., 2014 & Nature Genetics[4] \\
南极蠓 & Belgica Antarctica & $99 \mathrm{Mb}$ & Kelley et al., 2014 & Nature Communications[5] \\
帝企我 & Aptenodytes forsteri & $1.26 \mathrm{~Gb}$ & & GigaScience[6] \\
阿德利企找 & Pygoscelis adeliae & $1.23 \mathrm{~Gb}$ & Li et al., 2014 & Nature[7] \\
大西洋鲑鱼 & Salmo salar & $2.97 \mathrm{~Gb}$ & Lien et al., 2016 & GigaScience[8] \\
扁嘴副带腭鱼 & Parachaenichthys charcoti & $795 \mathrm{Mb}$ & Ahn et al., 2017 & Genes[9] \\
白鲸 & Delphinapterus leucas & $2.32 \mathrm{~Gb}$ & Jones et al., 2017 & GigaScience[10] \\
南极桡足类 & Tigriopus kingsejongensis & $295 \mathrm{Mb}$ & Kang et al., 2017 & Plos One[11] \\
北极红点鲑 & Salvelinus alpinus & $2.2 \mathrm{~Gb}$ & Christensen et al., 2018 & GigaScience[12] \\
南极美露鳕 & Dissostichus mawsoni & $824 \mathrm{Mb}$ & Chen et al., 2019 & \\
\hline
\end{tabular}




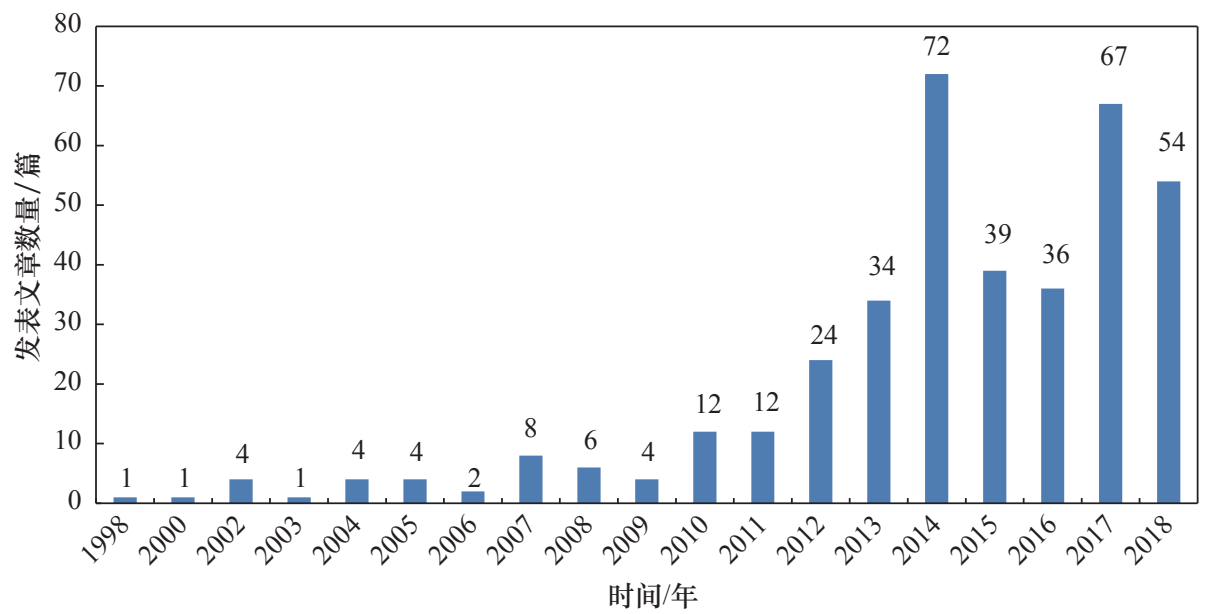

(a)

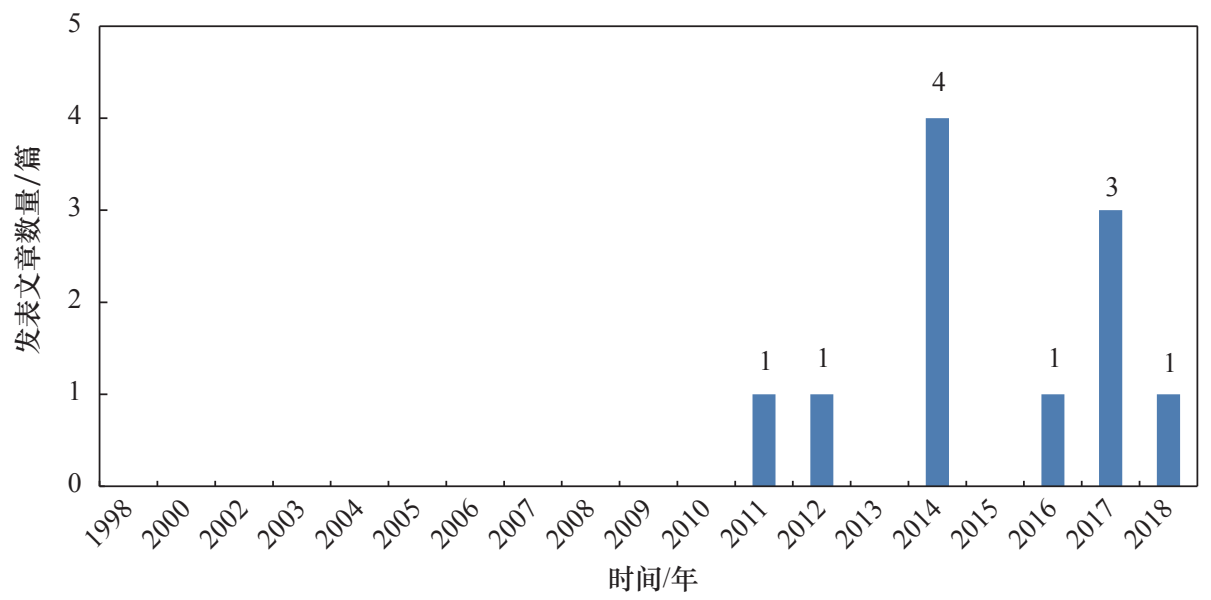

(b)

图 1 动物基因组发表文章数量（a）和极地动物基因组发表文章数量（b）

工具, 开展转录组分析, 挖掘功能基因, 为产业化 利用提供基因资源, 具有重要意义和应用潜力。据 不完全统计, 人们已对 31 种极地动物进行了转录 组测序, 其中北极物种有 8 个、南极物种有 23 个。 自 2008 年起，相关文章陆续发表; 2013 年文章发 表数量明显增多，2015 年达到小高峰。此类研究大 多在北美和欧洲机构中展开, 英美发表文章占 $45 \%$, 2012 年以来, 韩国的研究成果增长显著 (见图 2)。

\section{1. 生物对环境适应性的转录组研究}

极地以其极端环境引起人们对生物在极端环境 下生存机理的研究兴趣。研究主要集中在低温适应、 干燥胁迫、海水酸化、海洋变暖等方面。从植物 (如 南极嗜寒衣藻 [13]) 、无脊椎动物 (如南极线虫 [14] 、 南极水蚤 [15]）到鱼类（如博氏南冰鰧 [16]、南极 美露鳕 [17]、独角雪冰鱼 [18]、裸身雅南极鱼 [19]), 学者们对转录组进行了测序、组装, 对信号通路进
行分析, 研究这些物种对冷环境适应的分子机理。

Cocca 等 [20] 通过 cDNA 文库等手段, 发现 南极冰鱼发生了血红蛋白基因的丢失，导致血液呈 现纯白色; 随后研究发现在其中的 6 个物种中肌 红蛋白基因也跟着丢失, 使得心脏等器官也呈现白 色; 血红蛋白基因丢失使得冰鱼对氧气的运输能力 只有 $10 \%$, 可通过皮肤呼吸和心脏变大弥补这种缺 失 [21]。Xu 等 [22] 通过转录组分析进一步揭示冰 鱼通过血红蛋白基因丢失适应低温的分子机制。

Shin 等 [23] 通过对革首南极鱼、头带冰鱼、南 极多线鱼这 3 种南极鱼与生活在温带气候的鱼类的 转录组比较后发现, 南极鱼类的泛素结合蛋白表达 量较高, 而这类蛋白对寒冷环境下鱼体内蛋白保持 活性状态起重要作用。

Bilyk 等 [16] 发现泛素蛋白连接酶活性和蛋白 泛素化等途径相关的基因对博氏南冰膡在寒冷环境 


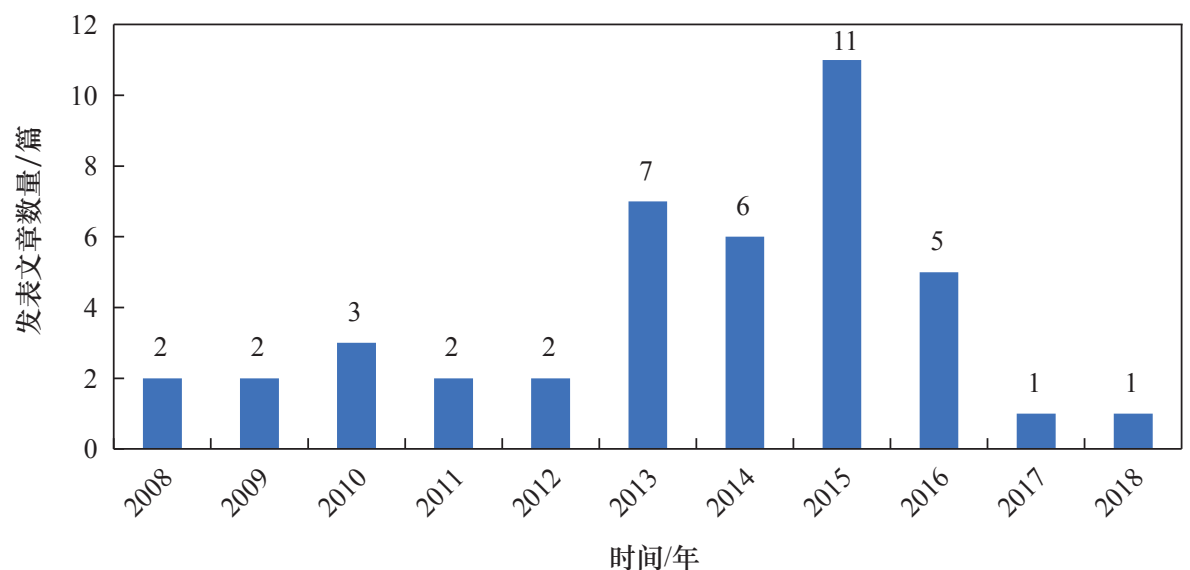

(a)

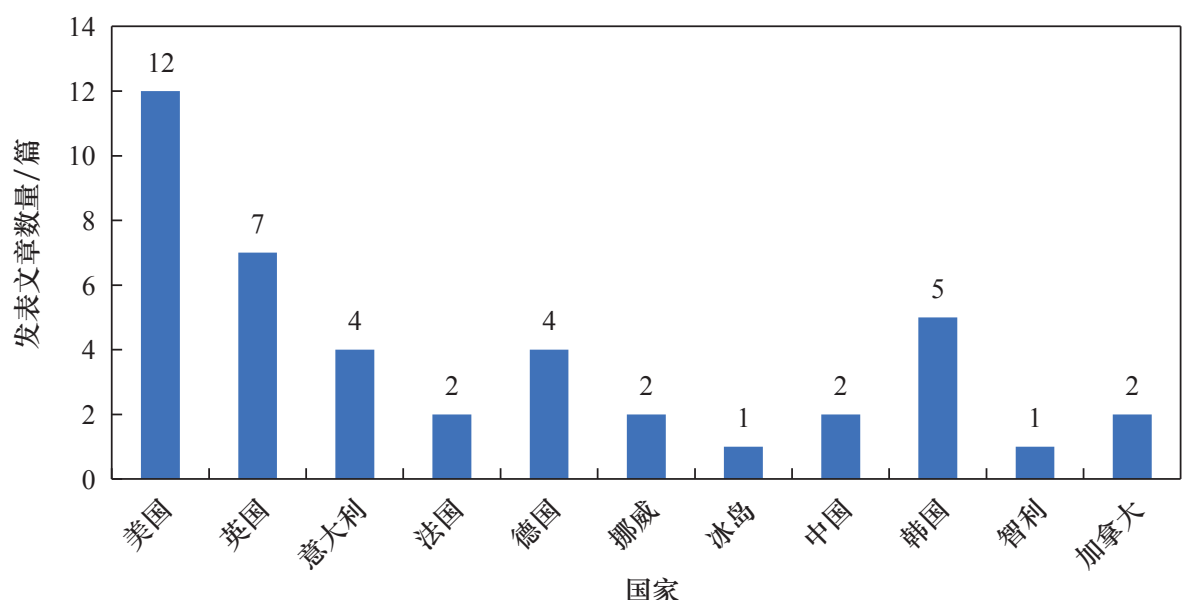

(b)

图 2 2008一-2018 年极地动物转录组发表文章数量（a）和发表的国家统计（b）

中的生存起到了重要作用。Coppe 等 [24] 发现独角 雪冰鱼在线粒体生物合成和有氧呼吸过程中, 鱼体 一直保持着基因复制和较高的线粒体密度，这或许 是独角雪冰鱼在寒冷环境并缺乏氧运输蛋白的情况 下，调节自身产能方式和效率，以适应外界环境的 一种方式。

全球气候变化使人们对两极动植物的生存问题 产生更多关注。海水酸化、气温升高是学者研究的 热点。Clark 等 [25] 发现, 在南极磷虾转录组中热 激蛋白 (Hsp70、Hsp90)、铁蛋白、谷胱甘肽 S 转 移酶（GST）的表达量较高, 可能在磷虾适应环境 变化中起重要作用。Meyer 等 [26] 通过转录组测序 研究了 $\mathrm{CO}_{2}$ 含量变化对磷虾的影响以及磷虾对环境 变化的适应性。Buckley 等 [27] 报道了环境温度升 高过程中伯氏肩孔南极鱼的转录水平上的变化, 发 现热激蛋白表达量并未有差异, 而其他与细胞应激 反应相关的基因表达量上调, 说明在全球变暖下伯
氏肩孔南极鱼对环境的适应性较差。Huth 等 [28] 则报道了伯氏肩孔南极鱼在海水酸化和气温升高的 胁迫下, 参与代谢转移、DNA 损伤修复、免疫系统、 细胞调亡通路的活跃性、细胞分化调控等途径的基 因表达量均较高。

\section{2. 生物对污染物应激反应的转录组研究}

Andersen 等 [29] 研究表明, 原油污染会造成成 纤维细胞生长因子基因（FGF7）的表达量大幅上 调, 对胆汁富集毒素鱼体的肝脏保护起到重要作用, 因此 FGF7 可作为生物体内监控原油泄漏的标志。 Kang 等 [30] 对污染物处理的南极隐须钩虾进行了 转录组测序, 篮选到 658 个基因可作为潜在的多氯 化联苯污染标志基因、168 个基因为潜在的全氟辛 烷磺酸污染标志基因、367 个基因为全氟辛酸污染 标志基因。Rhee 等 [31] 以北极绿海胆为材料, 研 究了多氯联苯对生物体的影响。发现在使用多氯联 苯处理北极绿海胆 $48 \mathrm{~h}$ 后, 其 11 个基因的表达量 
大幅上调，因此这些基因或许可以作为标志物，用 于环境中多氯联苯的检测和环境毒理学研究。

3. 生物发育阶段或不同组织的转录组分析

极地生物的生长发育也是学者们关注的重 点。自 Pitta 等 [32] 于 2008 年首次报道了磷虾 不同组织的转录组数据后, Seear 等 [33] 篎选出 磷虾蜕壳过程中的 26 个差异表达基因, 分别参 与几丁质合成、分化和吸收。海蛇尾的断肢可 以再生, 而南极海蛇尾的断肢再生速度是最慢 的。Burns 等 [34] 通过对南极海蛇尾转录组的分 析, 发现 $\mathrm{Wnt} / \beta$-catenin 信号通路、同源盒基因 (Hox) 及 $\mathrm{SRY}$ 盒-SOX 基因家族、 $\mathrm{TGF}-\beta$ 信号转 导通路可能在其断肢再生过程中发挥重要作用。 Gudbrandsson 等 [35] 对不同群体的北极红点鲑的 四个胚胎发育阶段进行转录组分析, 发现若干生物 途径的基因在胚胎发育过程中的表达量有差异，而 能量代谢和血液凝结相关基因在不同群体的鲑鱼 中差异表达; 单核苷酸多态性 (SNP) 频率分析 表明, 不同群体的鲑鱼之间存在大范围遗传分化。 Magnanou 等 [36] 对北极红点鲑不同组织的转录组 进行测序，分析了神经内分泌、代谢、行为等关键 生物学途径。

4. 极地动物功能基因的研究现状

通过同源克隆、基因组和转录组测序, 获得
一些极地动物的功能基因。据不完全统计，涉及到 30 多个物种, 其中 20 多个是鱼类; 同时涉及 20 多 个生命过程或功能（见表 2) [37 50]。

极地地区的一些鱼类具有合成抗冻蛋白的能 力, 以适应极地的低温生活条件。在断线真狼绵鳚 基因中，共发现抗冻蛋白 III 型有 7 个不同的变体, 翻译成 5 个不同的蛋白亚型; 亚型的分化是正向选 择的结果, 说明抗冻蛋白的多样性对生物体抗低温 有重要作用 [37]。南极多线鱼和南极鳕鱼中的抗冻 蛋白 IV 型基因序列相似度达 94\%, 进化分析显示 这个基因在硬骨鱼中广泛存在，并且内含子区域高 度保守。两个重组蛋白中均结出星型冰晶, 且冰晶 的温度在 $0.08{ }^{\circ} \mathrm{C}$, 证明了重组蛋白具有抗冻活性。

热休克蛋白 (HSPs) 是一类广泛存在的热应激 蛋白, 当生物体处于高温暴露时, 就会由热激发合 成此种蛋白, 以保护自身。在 $10{ }^{\circ} \mathrm{C}$ 的环境下, 南 极帽贝 HSP70 表达量可以显著增高, 表明 HSP70 在南极帽贝调节适应环境温度的过程中起到了重要 作用 [44]。在伯氏肩孔南极鱼、博氏南冰鰧、断线 真狼绵鳚这 3 种南极鱼中, HSC71 和 HSP70 的表 达量随低温处理时间增加而变化。研究表明, 在低 温环境下, 蛋白会发生冷变性或折叠错误, 导致分 子伴侣热击蛋白的表达量升高, 从而帮助生物体在 低温环境下生存 [45]。

表 2 已研究的功能基因（不完全统计）

\begin{tabular}{lccc}
\hline 功能基因 (蛋白) & 中文名 & 拉丁名 & 发表年份 \\
\hline 抗冻蛋白 & 断线真狼绵鳚 & Lycodichthys dearborni & $2010[37]$ \\
& 南极美露鳕 & Dissostichus mawsoni & $2011[38]$ \\
& 南极多线鱼 & Pleuragramma antarcticum & $2011[39]$ \\
& 南极鳕 & Notothenia coriiceps & $2011[39]$ \\
卵壳蛋白 & 南极鱼 & Notothenioids & $2016[40]$ \\
免疫球蛋白 & 伯氏肩孔南极鱼 & Trematomus bernacchii & $2002[41]$ \\
Tc 样转位子 & 独角雪冰鱼 & Chionodraco hamatus & $2002[42]$ \\
肌红蛋白 & 头带冰鱼 & Chaenocephalus aceratus & $2003[43]$ \\
热休克蛋白 & 南极帽贝 & Laternula elliptica & $2009[44]$ \\
& 伯氏肩孔南极鱼 & Trematomus bernacchii & $2005[45]$ \\
& 博氏南冰䲢 & Pagothenia borchgrevinki & $2005[45]$ \\
& 真裸南极鱼 & Harpagifer antarcticus & $2008[46]$ \\
芳香烃受体 & 北极红点鲑 & Salvelinus alpinus & $2015[47]$ \\
糖皮质激素受体 & 北极红点鲑 & Salvelinus alpinus & $2004[48]$ \\
红视蛋白基因 & 五种南极鱼 & - & $2012[49]$ \\
肌球蛋白 & 南极冰鱼 & Channichthyidae & $2011[50]$ \\
\hline
\end{tabular}




\section{三、问题与挑战}

\section{（一）极地动物基因组解析工作处于起步阶段}

受限于样品的获取技术, 目前只有 13 种极地 动物完成了全基因组测序, 占已进行基因组测序并 发表文章的动物种类 (380 余种) 的 3\%。相比而言, 极地动物基因组测序开展的规模小、种类少, 这与 极地相对丰富的动物资源相比远远不够。而且已测 序的极地动物中也主要以鱼类为主 (6 种), 还有许 多极地动物（如大西洋庸鲽、南极磷虾）的基因组 图谱尚未见报道。因此, 极地动物基因组研究有着 极大的发展潜力。

\section{（二）极地动物转录组测序缺乏系统性}

据不完全统计, 进行转录组测序的极地动物达 31 种, 但由于样品获取和保存技术等因素的限制, 转录组方面的研究比较零散, 难以系统支撑极地生 物特殊性状的遗传解析工作。因此, 对同一物种的 样品采集进行细化以及对其他极地物种展开批量化 转录组研究, 是规模化发掘极地动物基因资源的关 键, 也是进行极地动物特殊性状解析的基础。

\section{（三）功能基因的研究不够深入}

目前在多种极地动物中初步开展了一些功能基 因的研究, 但研究手段较单一, 主要以描述性分析 为主 (如克隆基因序列、表达模式分析等), 加之 模拟极地生存环境难度较大, 很难开发出极地模式 生物进行研究, 也没有相应细胞系等研究材料。这 导致在极地动物中缺乏相应的基因功能分析平台, 难以对基因功能进行验证和深入研究。

\section{（四）极地动物表观遗传研究有待开展}

表观遗传在动物适应外界环境和温度变化中起 着重要作用, 而极地的寒冷气候对极地动物表型影 响的表观遗传调控机制研究目前尚未见报道, 亟待 开展。

\section{（五）极地动物基因应用很少}

由于极地动物基因在获取、鉴定和功能分析方 面的限制, 加上缺少极地动物基因工程产品的研制, 迄今极地动物功能基因的应用还很薄弱, 基因工程 产品亟待研发。

\section{四、极地动物基因资源发掘与应用研究的技 术需求}

(一) 系统开展极地动物组学研究, 针对极地渔业 生物特殊性状展开遗传解析

系统开展极地动物组学研究, 研发极地动物样 品获取和保存技术。加大以极地渔业动物为主的基 因组测序研究, 有针对性地选取对极端环境适应等 具有特殊意义的极地动物展开组学研究, 为开展极 地动物适应性机制和全球环境变化影响等研究提供 理论依据。

\section{（二）构建极地动物基因功能研究平台, 对特有基 因功能进行深入研究}

进行极地动物特殊性状的遗传解析, 篮选关键 的功能基因, 建立极地动物细胞系, 探索极地动物 的实验室养殖技术, 开发极地渔业模式动物, 为极 地渔业动物基因功能验证分析提供技术保障。

\section{（三）开展极地动物适应极地生存环境的机制解析}

极地动物特殊的适应性为研究环境与基因互作 最终形成特定表型提供了理想材料。在极地动物中 开展表观遗传学研究, 有助于分析极地极寒环境对 极地动物基因表达的调控机制。极地鱼类一般生长 代谢缓慢、生命周期长, 但是也有一些鱼类生长速 度不亚于常温下的鱼类。例如大西洋庸鲽, 作为冷 水性鱼类, 耐低温能力强, 生长速度快, 2 年内可 从 $0.25 \mathrm{~kg}$ 长到 $2.5 \mathrm{~kg}$ 。对大西洋庸鲽的全基因组测 序和精细图谱绘制, 有助于解析大西洋庸鲽生长代 谢的分子机制, 可为其他鲠鲽鱼类和海水鱼类的养 殖提供创新思路和基因资源。

\section{（四）加强极地动物基因工程产品研发和应用研究}

基因组研究的最终目的就是开发出能够应用 的基因, 研制基因工程产品。以抗冻蛋白为例, 已广泛应用于食品添加剂、作物抗寒等领域, 但 现在的主要来源是提取天然抗冻蛋白, 产量受到 极大限制; 而通过基因工程技术则可以规模化生 产抗冻蛋白, 实现抗冻蛋白在细胞和组织冷冻保 存、食品、医药、化妆品等领域的产业化应用。 此外, 开展南极鱼类低温酶基因工程产品的研发, 开发其作为洗衣粉添加剂、消毒剂的应用途径也 
具有重要意义和应用前景。

\section{五、对策建议}

\section{（一）发展目标（2025 年和 2035 年）}

到 2025 年，完成 5 10 种极地鱼类和南极磷虾 等重要极地渔业生物的全基因组测序, 揭示重要极 地渔业动物适应特殊生存环境的分子机制; 在模式 鱼类或鱼类细胞系中通过基因编辑技术对极地鱼类 候选基因的功能进行验证; 探索适于极地动物暂 养的实验室条件, 为建立极地动物功能验证平台 提供支撑。

到 2035 年, 建立较为完善的极地动物基因资源 发掘平台, 完成 20 种以上极地渔业动物基因组解析; 完成 3 5 个极地渔业动物特殊性状的遗传解析计划, 鉴定一批在极地生存环境适应过程中的关键基因; 完善极地动物细胞系建立技术; 实现极地动物的实 验室暂养和长期培养, 应用于功能基因的深入研究; 挑选合适的候选基因, 研发 1 2 种极地渔业动物的 基因工程产品, 进行成果转化和产业应用。

\section{（二）重点任务}

\section{1. 极地渔业动物特殊性状的遗传解析}

加强对极地重要渔业动物基因组和转录组研 究, 增加测序的物种种类, 有针对性地对特殊性状 展开遗传解析; 开展极地渔业动物表观遗传学及其 调控动物适应寒冷机制的研究; 作为对生态系统和 产物资源都具有战略意义的物种，南极磷虾和大西 洋庸鲽等极地渔业动物基因组的精细图谱值得开展 系统、细致的绘制。

\section{2. 极地动物特有基因的功能验证}

深入挖掘和分析极地动物的基因组和转录组, 篮选极地动物特有基因（或进化出特殊功能的基 因）; 在模式鱼类或鱼类细胞系中，利用基因编辑 技术、过表达技术、转基因技术，对基因功能进行 验证和研究; 探索极地动物的实验室培养条件, 建 立极地动物基因功能研究平台。

3. 极地渔业动物基因工程产品的研发和应用

在极地动物抗寒、抗病、抗癌、快速生长、长 寿等方面, 针对已验证功能的基因, 通过基因工程 技术获得抗冻蛋白、低温酶、快速生长等基因工程 产物, 探索其在食品、医药、日用、水产养殖等领
域的应用潜力, 实现基因资源的成果转化和利用, 为这些领域实现增值提效提供基因资源和产品。

\section{（三）重大科技工程建议}

建议设立极地渔业生物基因资源发掘与应用的 国家重点研发计划专项，重点支持如下方向：(1)极 地鱼类和南极磷虾等极地重要渔业动物全基因组测 序及精细图谱绘制； (2)极地渔业动物特殊性状的遗 传解析； (3)极地渔业动物细胞培养与细胞系建立; (4)极地渔业动物基因工程产品研发与应用。

\section{参考文献}

[1] Star B, Nederbragt A J, Jentoft S, et al. The genome sequence of Atlantic cod reveals a unique immune system [J]. Nature, 2011, 477(7363): 207-210.

[2] Miller W, Schuster S C, Welch A J, et al. Polar and brown bear genomes reveal ancient admixture and demographic footprints of past climate change $[\mathrm{J}]$. Proceedings of the National Academy of Sciences of the United States of America. 2012, 109(36): E23822390.

[3] Shin S C, Ahn D H, Kim S J C, et al. The genome sequence of the Antarctic bullhead notothen reveals evolutionary adaptations to a cold environment [J]. Genome Biololgy, 2014, 15(9): 468.

[4] Yim H S, Cho Y S, Guang X, et al, Minke whale genome and aquatic adaptation in cetaceans [J]. Nature Genetics, 2014, 46(1): 88-92.

[5] Kelley J L, Peyton J T, Fiston-Lavier A S, et al, Compact genome of the Antarctic midge is likely an adaptation to an extreme environment [J]. Nature Communications, 2014, 5: 4611.

[6] Li C, Zhang Y, Li J, et al. Two Antarctic penguin genomes reveal insights into their evolutionary history and molecular changes related to the Antarctic environment [J]. GigaScience, 2014, 3(1): 27.

[7] Lien S, Koop B F, Sandve S R, et al. The Atlantic salmon genome provides insights into rediploidization [J]. Nature, 2016, 533(7602): 200-205

[8] Ahn D H, Shin S C, Kim B M, et al. Draft genome of the Antarctic dragonfish, Parachaenichthys charcoti [J]. GigaScience, 2017, 6(8): 1-6.

[9] Jones S J M, Taylor G A, Chan S, et al. The genome of the Beluga whale (Delphinapterus leucas) [J]. Genes (Basel), 2017, 8(12): Pii:E378.

[10] Kang S, Ahn D H, Lee J H, et al, The genome of the Antarctic-endemic copepod, Tigriopus kingsejongensis [J]. GigaScience, 2017, 6(1): $1-9$

[11] Christensen K A, Rondeau E B, Minkley D R, et al. The Arctic charr (Salvelinus alpinus) genome and transcriptome assembly [J]. Plos One, 2018, 13(9): e0204076.

[12] Chen L B , Lu Y, Li W H, et al. The genomic basis for colonizing the freezing Southern Ocean revealed by Antarctic toothfish and Patagonian robalo genomes [J]. GigaScience, 2019, 8: 1-16. 
[13] Liu C L, Huang X H. Transcriptome-wide analysis of DEADbox RNA helicase gene family in an Antarctic psychrophilic alga Chlamydomonas sp ICE-L [J]. Extremophiles, 2015, 19(5): 921-931.

[14] Thorne M A S, Kagoshima H, Clark M S, et al. Molecular analysis of the Cold Tolerant Antarctic Nematode, Panagrolaimus davidi [J]. Plos One, 2014, 9(8): e104526.

[15] Kim H S, Lee B Y, Han J, et al. De novo assembly and annotation of the Antarctic copepod (Tigriopus kingsejongensis) transcriptome [J]. Marine Genomics, 2016, 28: 37.

[16] Bilyk K T, Cheng C H C. Model of gene expression in extreme cold-reference transcriptome for the high-Antarctic cryopelagic notothenioid fish Pagothenia borchgrevinki [J]. BMC Genomics, 2013, 14: 634.

[17] Chen Z, Cheng C H C, Zhang J, et al. Transcriptomic and genomic evolution under constant cold in Antarctic notothenioid fish [J]. Proceedings of the National Academy of Sciences of the United States of America, 2008, 105(35): 12944.

[18] Coppe A, Agostini C, Marino I A M, et al. Genome evolution in the cold: Antarctic icefish muscle transcriptome reveals selective duplications increasing mitochondrial function [J]. Genome Biology and Evolution, 2012, 5 (1): 45.

[19] Papetti C, Harms L, Windisch H S, et al. A first insight into the spleen transcriptome of the notothenioid fish Lepidonotothen nudifrons: Resource description and functional overview [J]. Marine Genomics, 2015, 24: 237.

[20] Cocca E, Ratnayake-lecamwasam M, Parker S K, et al. Genomic remnants of alpha-globin genes in the hemoglobinless antarctic icefishes [J]. Proceedings of the National Academy of Sciences of the United States of America, 1995, 92: 1817-1821.

[21] O'Brien K M, Mueller I A. The unique mitochondrial form and function of Antarctic channichthyid icefishes [J]. Integrative and Comparative Biology, 2010, 50: 993-1008.

[22] Xu Q, Cai C, Hu X, et al. Evolutionary suppression of erythropoiesis via the modulation of TGF- $\beta$ signalling in an Antarctic icefish [J]. Molecular Ecology, 2015, 24: 4664-4678.

[23] Shin S C, Kim S J, Lee J K, et al. Transcriptomics and comparative analysis of three Antarctic notothenioid fishes [J]. Plos One, 2012, 7(8): e43762.

[24] Coppe A, Agostini C, Marino I A, et al. Genome evolution in the cold: Antarctic icefish muscle transcriptome reveals selective duplications increasing mitochondrial function [J]. Genome Biology and Evolution, 2013, 5: 45-60.

[25] Clark M S, Thorne M A S, Toullec J Y, et al. Antarctic krill 454 pyrosequencing reveals chaperone and stress transcriptome $[\mathrm{J}]$. Plos One, 2011, 6(1): e15919.

[26] Meyer B, Martini P, Biscontin A, et al. Pyrosequencing and de novo assembly of Antarctic krill (Euphausia superba) transcriptome to study the adaptability of krill to climate-induced environmental changes [J]. Molecular Ecology Resource, 2015, 15: 6.

[27] Buckley B A, Somero G N. cDNA microarray analysis reveals the capacity of the cold-adapted Antarctic fish Trematomus bernacchii to alter gene expression in response to heat stress [J]. Polar Biology, 2009, 32 (3): 403.

[28] Huth T J, Place S P. Transcriptome wide analyses reveal a sustained cellular stress response in the gill tissue of Trematomus ber- nacchii after acclimation to multiple stressors [J]. BMC Genomics, 2016, 17: 127.

[29] Andersen Ø, Frantzen M, Rosland M, et al. Effects of crude oil exposure and elevated temperature on the liver transcriptome of polar cod (Boreogadus saida) [J]. Aquatic Toxicology, 2015, 165: 9.

[30] Kang S, Kim S, Park H. Transcriptome of the Antarctic amphipod Gondogeneia antarctica and its response to pollutant exposure [J]. Marine Genomics, 2015, 24: 253.

[31] Rhee J S R, Kim B M, Choi B S, et al. Transcriptome information of the Arctic green sea urchin and its use in environmental monitoring [J]. Polar Biology, 2014, 37 (8): 1133.

[32] De Pittà C, Bertolucci C, Mazzotta G M, et al. Systematic sequencing of mRNA from the Antarctic krill (Euphausia superba) and first tissue specific transcriptional signature [J]. BMC Genomics, 2008, 9: 45.

[33] Seear P J, Tarling G A, Burns G, et al. Differential gene expression during the moult cycle of Antarctic krill (Euphausia superba) [J]. BMC Genomics, 2010, 11: 582.

[34] Burns G, Thorndyke M C, Peck L S, et al. Transcriptome pyrosequencing of the Antarctic brittle star Ophionotus victoriae [J]. Marine Genomics, 2013, 9: 9.

[35] Gudbrandsson J, Ahi E P, Franzdottir S R, et al. The developmental transcriptome of contrasting Arctic charr (Salvelinus alpinus) morphs [J]. F1000Research, 2015, 4: 136.

[36] Magnanou E, Noirot C, Falcón J, et al. Sequencing and characterization of a multi-organ Arctic charr transcriptome: A toolbox for investigating polymorphism and seasonal life in a high Arctic fish [J]. Marine Genomics, 2016, 29: 45.

[37] Kelley J L, Aagaard J E, MacCoss M J, et al. Functional diversification and evolution of antifreeze proteins in the antarctic fish Lycodichthys dearborni [J]. Journal of Molecular Evolution, 2010, 71: 111-118.

[38] Nicodemus-Johnson J, Silic S, Ghigliotti L, et al. Assembly of the antifreeze glycoprotein/trypsinogen-like protease genomic locus in the Antarctic toothfish Dissostichus mawsoni (Norman) [J]. Genomics, 2011, 98(3): 194-201.

[39] Lee J K, Kim Y J, Park K S, et al. Molecular and comparative analyses of type IV antifreeze proteins (AFPIVs) from two Antarctic fishes, Pleuragramma antarcticum and Notothenia coriiceps $[\mathrm{J}]$. Comparative Biochemistry and Physiology B-Biochemisty \& Molecular Biology, 2011, 159(4): 197-205.

[40] Cao L X, Huang Q, Wu Z C, et al. Neofunctionalization of zona pellucida proteins enhances freeze-prevention in the eggs of Antarctic notothenioids [J]. Nature Communications, 2016, 7(1): 12987.

[41] Oreste U, Coscia M. Specific features of immunoglobulin VH genes of the Antarctic teleost Trematomus bernacchii [J]. Gene, 2002, 295(2): 199-204.

[42] Capriglione T, Odierna G, Caputo V, et al. Characterization of a Tc1-like transposon in the Antarctic ice-fish, Chionodraco hamatus [J]. Gene, 2002, 295(2): 193-198.

[43] Small D J, Moylan T, Vayda M E, et al. The myoglobin gene of the Antarctic icefish, Chaenocephalus aceratus, contains a duplicated TATAAAA sequence that interferes with transcription [J]. Journal of Experimental Biology, 2003, 206: 131-139. 
[44] Kim M, Ahn I Y, Kim H J, et al. Molecular characterization and induction of heat shock protein 90 in the Antarctic bivalve Laternula elliptica [J]. Cell Stress \& Chaperons, 2009, 14(4): 363-370.

[45] Place S P, Hofmann G E. Constitutive expression of a stressinducible heat shock protein gene, HSP70, in phylogenetically distant Antarctic fish [J]. Polar Biology, 2005, 28(4): 261-267.

[46] Clark M S, Fraser K P P, Burns G, et al. The HSP70 heat shock response in the Antarctic fish Harpagifer antarcticus [J]. Polar Biology, 2008, 31(2): 171-180.

[47] Ahi E P, Steinhäuser S S, Pálsson A, et al. Differential expression of the aryl hydrocarbon receptor pathway associates with craniofacial polymorphism in sympatric Arctic charr [J]. EvoDevo,
2015, 6: 27.

[48] Aluru N, Jorgensen E H, Maule A G, et al. PCB disruption of the hypothalamus-pituitary-interrenal axis involves brain glucocorticoid receptor downregulation in anadromous Arctic charr [J]. American Journal of Physiology-Regulatory Integrative and Comparative Physiology, 2004, 287(4): R787-793.

[49] Miyazaki T, Iwami T. Molecular cloning of cDNA encoding red opsin gene in the retinas of five Antarctic notothenioid fishes [J]. Polar Biology, 2012, 35(5): 775-783.

[50] Borley K A, Sidell B D. Evolution of the myoglobin gene in Antarctic icefishes (Channichthyidae) [J]. Polar Biology, 2011, 34(5): 659-665. 